\title{
Systemic Reform Efforts in the U.S.
}

\author{
Role of Information Technology in Fostering Collaboration \\ within New Partnerships
}

Christopher A. Thorn

University of Wisconsin-Madison - Wisconsin Center for Education Research cathom@wisc.edu

Abstract: While data-based decision making and decision support systems are making inroads into educational management, the human capacity to evaluate programs, curricula, and other reform efforts has not kept pace. The latest generation of collaborative systems that support knowledge exchange and expertise location services hold great promise for building communities around these issues and supporting emerging research capacity in schools and districts.

Key words: Collaboration, knowledge management systems, school reform.

\section{INTRODUCTION}

Much of the work being done on the roll of information technology in improving school performance is in the area of data-based or data-driven decision making. The rising importance of standardized test results in national-, state-, and local-level accountability has led to a new interest in using individual level data generated for external reporting to guide local improvement efforts. The concept of school and district report cards has been taken to the next level in many places with interactive web sites that allow external viewers to examine the performance of subgroups and different accountability metrics. Data warehouse efforts within districts are attempts to put more instructionally relevant data into the hands of decision makers at all levels. 
What these efforts often neglect to acknowledge is that we already had a pretty good idea of which groups are suffering from performance deficits. Improvement efforts have been the focus of many years of professional development and curriculum reform. The U.S. National Science Foundation has initiated a program area that combines lessons learned from a decade of systemic reform initiatives and from recent advances in research-based teaching materials for K-12 education in STEM (Science, Technology, Engineering, and Math). This new program is called Math and Science Partnerships and is intended to help bridge the gaps between state of the art research in STEM, leading education schools, and districts willing to adopt high-strength curricula and changes in teaching practices directed at closing the achievement gap between white and minority (particularly African American) students.

\section{RESEARCH AGENDA}

The research effort here is focused on the question of what support is needed to build human capacity to use student and staff outcome data. Early efforts to support data-based decision making were founded on a philosophy that could be summed up as "build it, and they will come". While there is little argument that school data systems were inadequate to support good decision making based on performance measures (for student or staff), the creation of these systems did not imbue decision makers with skills they did not possess. This approach also did nothing to address the need to build social networks around evidence to promote the diffusion and use of successful strategies. This gap has led my research and development work in the direction of examining the role of collaborative (primarily web-based) technologies in providing the social space to build these communities and the related skills.

The program I will be describing as a backdrop for this paper is the System-wide Change for All Learners and Educators [SCALE]. The SCALE partnership is made up of two universities and four school districts. First, there are two large university research centers - the Wisconsin Center for Education Research [WCER], University of Wisconsin-Madison and the Learning Research and Development Center [LRDC], University of Pittsburgh. Both of these partners bring different strengths to the partnership. WCER connects the partnership to the $\$ 600$ million a year research base at UW-Madison - including leading centers for materials science, genetics, computers science, mathematics, and engineering. The LRDC has a outreach arm (the Institute for Learning [IfL]) that provides high-level technical assistance to 12 medium and large school districts across the country. Its 
approach, known as disciplinary literacy, is a holistic approach to teaching reform and related governance structures that is closely tied to the research base at the LRDC. This university partnership is supporting the development of new immersion curricula units in STEM subject areas that has as its goal the opportunity for each student to have one or more experiences each year doing authentic, hands on science. The SCALE partnership at the district level is made of two large districts - Los Angeles Unified School District and Denver Public Schools - and two medium-sized districts - Madison Metropolitan School District and Providence School District.

There are several key differences in this new round of funded projects that makes this partnership different from previous systemic reform efforts. First, SCALE is not trying to create new teacher training and teacher professional development relationships with these districts. The IfL already has existing, strong ties to each district. Each district has already bought into the professional development strategies of the IfL and is working with local coordinators to adapt the IfL approach to local district standards and organizational structures.

Second, UW-Madison is home to a number of large research efforts that have at least a decade of experience developing K-12 materials as a part of their STEM research efforts. Madison also has ties to similar projects at other large research universities and can act as a clearing house for similar work from other research teams.

Finally, the National Science Foundation has with its most recent round of large grants ( $\$ 35$ million for five years), operationalized a new organizational model for research coordination. In the past, grants would have been made at this amount or less. Any university coordination or interaction with more than one or two districts would have gone through relevant program officers at the National Science Foundation. The MSP program recognizes that coordination and cooperation at this level cannot be accomplished with the NSF program officers as the bottleneck for information flow. In a sense, the NSF has delegated part of its management authority to the partnership. In fact, the governing agreement for the partnership is not a traditional contract. It is called a cooperative agreement and is negotiated after the award is made. The negotiation process both allowed the NSF officials to help refine the goals and gave the partners a clearer picture of what the metrics of success would be.

\section{A CASE STUDY OF SYSTEM IMPLEMENTATION}

The primary study in the project is a case study of the implementation of an enterprise-level knowledge management and expertise discovery system 
across the leadership level of this partnership. As I said at the outset, there is a strong focus - particularly at the district level - on the use of achievement data (both high stakes outcome data and interim measures) to track the performance of states, districts, schools, teachers, and children. From the perspective of SCALE, this approach is clearly important. The partners must be able to evaluate the effectiveness of the interventions and both intermediate test scores and high stakes results will provide much of the key data for that work. However, there is a larger and more challenging problem on the other side of the partnership. The university units, school district offices, and funders do not share a single security environment and lack the tools necessary to support the complex interactions that would be necessary for them to achieve their goals. At this point, it is important to consider the characteristics of the partners from the point of view of their traditional strengths and ways of doing business.

The SCALE partnership is made up of two distinctly different groups. The organizational characteristics of these groups suggest that different forms of interaction will be differentially effective and that incentives for engagement will have to reflect each member's particular needs. In the case of the R\&D units - WCER and LRDC - the two units are engaged in research into the basic elements of educational sciences. We also see both groups using that research expertise to gain a competitive advantage in the market of ideas (academic press, recruitment of faculty, getting research grants, etc.). There are areas in which two centers compete directly in the same markets (NSF funding for math or systemic reform research) and areas in which we have very little overlapping interest such as the IfL's teacher professional development programs and its presence in districts.

The second cluster of organizations is the districts. The literature on collaboration would codify them more in the role of core customer in a close supply-chain relationship. The districts actually have little in common since they serve different markets and in no way compete with one another. There are no strong, near-term links that tie these organizations to one another aside from growing concerns about persistent gaps in test scores by race/ethnicity. The long-term prospect of leveraging of successful models may hold some appeal, but many districts find replication within their own districts to be enough of a challenge.

\subsection{Characteristics of Successful Cooperative Ventures}

In earlier (Thorn 1995) work, I have argued that successful collaboratives have to focus first on defining the overlapping interest that members share. One of the ways this condition is met in SCALE is the agreement on the common problems - high dropout rates, low test scores in math and science 
in urban districts, and widening performance gaps between whites and other ethnic/racial groups. This agreement on the challenge provides a shared sense of threatened core values - equal education for all, issues of competitiveness and success as a nation of large portions of the population are undereducated, social and economic decay of urban centers are a few of the core concerns shared by all members. This act of defining shared values and concerns both creates a social place for engaging in the partnership's work and says to everyone involved that other efforts by the members that do not fit with this common interest are not on the table for discussion.

The other important finding from this research was a short list of characteristics of successful consortia. First, there had to be a small leadership group that engaged in what is often called unconditional giving or (in economic terms) the commitment of resources beyond a level that appears rational for short- to mid-term calculations. Second, there had to be formal, transparent agreements about methods for the organization of work in the collaborative space. This included training around meeting management, conflict resolution, etc. This creation of a common working culture was a key factor in helping members from different organizations to be effective and productive after a short time. Members of one particularly successful consortium described the importance of knowing how to interact with others on his team and knowing they would all be interacting based on accepted norms and procedures. This certainty kept conflict to a minimum and helped to keep meetings short and productive. This contrast to the practices of their home organizations (computer and semiconductor firms) was quite striking for many members.

The creation of complex consortia of research units and school districts will require new workflows for creating curriculum and teacher training materials. It will require new tools for coordinating geographicallydistributed teams from different organizations. These new groupings will transcend traditional hierarchical relationships within each partner and will have no mechanisms for controlling the work of individual members. Rather, these new teams must be collaborative communities that focus on shared goals and that are motivated by those goals.

At the same time, each of the individual members of the collaborative will also be creating new teams within their respective organizations that will be directly responsible to leadership. These teams will likely cross existing organizational boundaries (discipline, sub-district, roles) and will need additional support for their work, since traditional supports would be based on the needs of their operational units and not reflect the needs of a new organization form. 


\subsection{A Comparison of Two Research-Based Systems}

What this problem space calls for is a set of collaboration tools that support group work and knowledge management in several forms. First, loose confederations of collaborators that are engaged in problem definition and in creating new communities will need tools that allow for the creation of new affinity groups. One of the problems encountered by teams of scientists as they begin coordination with science education and other teacher education professionals is that neither group has clear understanding of who should be working with whom. Tools such as the Fraunhofer Institute's Basic Support for Cooperative Work [BSCW] provide shared folders, discussion lists, tasks, and calendars for geographically-distributed teams.

An important aspect that is often overlooked, however, is its approach to creating groups. BSCW supports the traditional method of forming groups in IT systems. One can create predefined groups as a manager and invite all appropriate members to join. This group is typically assigned to a particular, pre-defined directory structure. Another option, however, is for individual users to invite others to their own work spaces to collaborate on smaller tasks. This identification of and support for local affinity groups is a powerful addition to collaborative technologies and enables partnerships that coalesce and flourish outside of traditional hierarchies.

This feature is a key difference between BSCW and many other collaborative environments that are only extensions/virtualizations of existing hierarchies. One of the strengths of BSCW in this area is a philosophy of making membership in the environment explicit and searchable. Openness is a default setting. While this design approach makes collaboration across teams within the same organization much easier, it also makes more complex forms of organization and the ability to hide groups that need protection from oversight or some form of anonymity very difficult to support. Sophisticated security models and support for complex organizational forms exist at the other end of the tool spectrum. This emerging need for enterprise-scale collaborative tools encouraged me to start a new research initiative to find and implement a collaborative environment designed around complex partnerships. This effort identified Intraspect ${ }^{1}$ as a leader in the field.

\section{FINDINGS}

While the initial research focused on BSCW, the ongoing work is focused on the creation of collaborative work and presentation spaces in our 
new Intraspect environment. We are now nine months into an implementation of this high-level collaboration, knowledge discovery, and expertise location system. My team is in the midst of documenting the affordances of the system for different forms of university-district-school partnerships. The preliminary finding show identify four different features that seem to be necessary to support the ongoing work across the different managerial levels of the partnership as well as vital to the work of dispersed teams working on particular curricular areas.

\subsection{Secure Space for Risk Taking is Vital in Districts with Low Trust between Groups}

Teachers or building leaders taking advantage of new high strength curricula or other professionally challenging endeavors may see student outcomes decline in the first year of implementation. In a high stakes environment, this can be seen as risky behavior. Participants need a safe space to collaborate that cannot be observed by others above them in the school or district hierarchy. At the same time, district leaders need to be able to provide political and other social support to teams at a distance - perhaps in a school climate that is not open to change. The ability to create a secure ad hoc space for collaboration can also free participants from some of the challenges of hierarchy.

\subsection{Building Ties to Experts (and Expert Knowledge)}

Intraspect and other KM systems can be seen as repositories for complex collections and may capture tacit knowledge in the form of email exchanges and other informal data types. One aspect of $\mathrm{KM}$ support in which Intraspect excels is the area of expertise identification. The metadata architecture and search engine preferences the association of individual with search terms. This makes it trivially easy to locate people associated with artifacts, concepts, or groups. When one searches, named users are always associated visually with all hits to make location easier. The search logic also preferences individuals in the system who are the owners of documents that contain the search terms. Multiple hits in documents introduced into the system by an individual will usually put the user in the top 10 hits. This linkage between artifacts and human expertise is a feature that SCALE is using explicitly to foster connections and knowledge exchange between groups. 


\subsection{Building Custom Spaces for Work and Presentation}

This complex partnership has multiple dimensions of membership organization, knowledge domain, level in the hierarchy, etc. The work of any individual member will differ based on their role in any grouping of actors. In the case of district leaders, they have asked for presentation spaces that display current and planned activities that will be taking place in their district as well as staff and student outcomes from past events. Disciplinary teams, on the other hand, want work spaces in which they can exchange and edit documents and manage complex workflows. Research and evaluation teams want workspaces organized around the projects deliverables. The object oriented design of the Intraspect system allows for the rapid development of custom spaces that explicitly support these different configurations and needs. No district- or university-centric system could provide these services. This enterprise/supply chain problem space requires specialized tools to address the challenges faced by the SCALE partnership.

\subsection{Object Oriented Design for Customization}

As noted above, object oriented technology has proven to be a crucial element of this implementation. The complexity of this research and development project with its tight links to school districts and their reform efforts means that we have many users who have multiple group memberships and may serve in multiple roles across those groups. Tools to support complex organizational models must respect this reality and provide quick and easy access to information and work spaces based on complex memberships.

\section{CONCLUSION}

The jury is still out on the effectiveness of the SCALE partnership. What seems clear, however, is the importance of providing collaborative support to university-district-school partnerships that allow for secure access to expertise and custom spaces. The National Science Foundation has reviewed the progress to date and is working with my group to explore extending our Intraspect environment to include members at the foundation. They have identified the lack of a collaborative environment as one of the biggest barriers they face in helping these large research projects deliver on their stated goals. 


\section{REFERENCES}

Thorn, C. (1995). State Involvement in the Semiconductor Industry: The Role and Importance of Consortia. Benevolent conspiracies: the role of enabling technologies in the welfare of nations : the cases of SDI, SEMATECH, and EUREKA. H. Willke, C. P. Krück and C. Thorn. New York, Walter de Gruyter.

1 Recently renamed Vignette Business Collaboration Server after the firm was purchased by Vignette Corporation in December 2003. 